\title{
Navigating the R Package Universe
}

\author{
by Julia Silge, John C. Nash, and Spencer Graves
}

\begin{abstract}
Today, the enormous number of contributed packages available to R users outstrips any given user's ability to understand how these packages work, their relative merits, or how they are related to each other. We organized a plenary session at useR!2017 in Brussels for the R community to think through these issues and ways forward. This session considered three key points of discussion. Users can navigate the universe of $R$ packages with (1) capabilities for directly searching for R packages, (2) guidance for which packages to use, e.g., from CRAN Task Views and other sources, and (3) access to common interfaces for alternative approaches to essentially the same problem.
\end{abstract}

\section{Introduction}

As of our writing, there are over 13,000 packages on CRAN. R users must approach this abundance of packages with effective strategies to find what they need and choose which packages to invest time in learning how to use. At useR!2017 in Brussels, we organized a plenary session on this issue, with three themes: search, guidance, and unification. Here, we summarize these important themes, the discussion in our community both at useR!2017 and in the intervening months, and where we can go from here.

Users need options to search R packages, perhaps the content of DESCRIPTION files, documentation files, or other components of R packages. One author (SG) has worked on the issue of searching for $\mathrm{R}$ functions from within $\mathrm{R}$ itself in the sos package (Graves et al., 2017). Other options have been built such as RDocumentation.org (Cornelissen, 2018).

Guidance about what package to use for any given task is available from multiple resources for users. R users can turn to long-established resources like CRAN Task Views (Zeileis, 2005), or newer options under current development such as packagemetrics (Firke et al., 2018) or the CRANsearcher RStudio addin (Krouse and Calatroni, 2017). One author (JS) organized a survey before useR about how $\mathrm{R}$ users learn about $\mathrm{R}$ packages that informed our discussion and is summarized here.

By unification, we largely mean meta-packages or wrappers, packages that call other, related packages for a common set of tasks. A unified wrapper package provides a common API through which to access many different implementations for a certain task. One author (JN) has been particularly involved in numerical optimization techniques and presented possibilities there and beyond. More generally, as revealed during breakout discussions at useR!2017 and afterward, there are opportunities to merge either packages or their functionality. Such ideas require human cooperation and some give and take in a realm where egos can take precedence over ease of use.

After our main presentation at useR!2017, we broke out into three smaller sessions focused on these themes. We are encouraged by the engaged attendance and vigorous participation from the community we experienced, and hope to use our community's enthusiasm and ideas to move forward with steps that will improve the value of the R ecosystem.

\section{Search}

There are a number of different search capabilities for $\mathrm{R}$, with different characteristics and strengths. The ways to search for help using $\mathrm{R}$ have proliferated in much the same way that the number of packages has, with some of the same challenges. It is not clear to users what the best way to search is, and many if not most users are not even aware of the search capabilities that have been built.

The R Project for Statistical Computing's website has had a page focused on "Getting Help with R" (R Core Team, a) from early in R's history. This official overview of various help facilities recognized by the R Core Team includes functions such as help(), demo(), apropos(), help. search(), and vignettes. This search and help functionality, used from $R$ itself, accesses only locally installed documentation. This page also currently points to resources such as the CRAN Task Views, FAQ pages, Stack Overflow, and $\mathrm{R}$ email lists.

Other search capabilities have been developed by the R community over the years, focused on different types of searching. The R Site Search built by Baron is one of the longest running, and can also be accessed from R itself using the RSi teSearch() function in utils (R Core Team, 2018) and the sos package. The sos: :findFn() function returns a data. frame of class findFn summarizing the help pages matching a search term. These objects can be combined by union and intersection, summarized by package, and output to an Excel file with sheets giving one row for each package and for each help 
Table 1: Percentage of respondents who chose each answer on survey

\begin{tabular}{ll}
\hline How do you currently discover and learn about R packages? & $\%$ of respondents \\
\hline Social media such as blogs, R-bloggers, Twitter, Slack, or GitHub contacts & $79.8 \%$ \\
General search websites such as Google and Yahoo & $57.0 \%$ \\
Your personal network, such as colleagues and professors & $41.6 \%$ \\
Books, textbooks, or journal articles (JSS, JOSS, R-Journal) & $31.9 \%$ \\
Conferences, meet-ups, or seminars & $24.1 \%$ \\
CRAN Task Views & $21.8 \%$ \\
Email lists such as r-help, r-packages, or r-pkg-devel & $15.3 \%$ \\
R-specific search websites such as METACRAN or Rdocumentation & $11.1 \%$ \\
Other & $4.2 \%$ \\
R packages built for search such as the sos package & $2.2 \%$ \\
\hline
\end{tabular}

page sorted by package.

The rseek site of Goodman (2007) is another resource that has been available for over a decade, and searches not only documentation but also GitHub issues, social media, and more. The R Package Documentation site of Howson (2018) is a newer option that includes not only a way to search R documentation but also the ability to run $\mathrm{R}$ in the browser. Another full-featured and modern website for search is RDocumentation.org, which offers users the ability to contribute examples.

Other options for search capability focus not on documentation or individual functions but on packages and package descriptions. The search capability at METACRAN (Csárdi and Ooms), crantastic (Wickham and Mæland), and the RStudio addin CRANsearcher allow users to search the descriptions and titles of packages to find tools that fit their analysis needs.

This discussion of search options for $\mathrm{R}$ users is intended to be thorough but not entirely exhaustive, and to demonstrate the variety of resources available. Many search sites are not clear how to use their search capabilities (single words vs. structured queries), and the algorithms used for ranking results give disparate results. An additional point to be considered is to what extent these options for search are open source, and what effect that could have, either positive or negative. For example, the source code for both METACRAN and CRANsearcher is entirely open on GitHub while RDocumentation.org is maintained by DataCamp, a privately held company.

The analysis in this section is summarized in a Wikiversity article (Graves, 2017); the article is on Wikiversity so anyone can contribute to it. At useR!2017, after the large contributed session, we broke out into three smaller sessions for discussion and brainstorming. In the breakout session focused on search, some participants commented that user reviews, available on at least crantastic among these existing resources, are useful alongside search. The discussion overall led to the development of a draft proposal for improving the ability of $\mathrm{R}$ users to search $\mathrm{R}$ packages, which is available on Wikiversity; anyone can contribute to moving it closer to realization (Graves, 2018). Overall, perhaps it is time for the R Project for Statistical Computing's "Search" (R Core Team, c) page to include more updated options.

\section{Guidance}

In preparation for this session, one author (JS) ran a brief online survey in the spring of 2017 to ask $\mathrm{R}$ users how they currently discover and learn about $\mathrm{R}$ packages. The results of this survey are available in an R package packagesurvey (Silge, 2018) on GitHub. There were 1039 respondents to this survey, which had a single multiple select question on it, "How do you currently discover and learn about $\mathrm{R}$ packages?"

Responses to this survey were fielded from $\mathrm{R}$ email help lists, local $\mathrm{R}$ meetup groups, social media such as Twitter, and affinity groups such as R-Ladies. Figure 1 shows when users responded to the survey. The respondents to this survey overwhelmingly look to social media including blogs and Twitter to learn about $\mathrm{R}$ packages, and also make use of general search sites and their personal network.

There were helpful, insightful answers from people contributing to the "other" option. R users use Stack Overflow to learn about R packages, as well as options like CRANberries (Eddelbuettel) and crantastic, both of which have RSS feeds that users follow. Other users mentioned learning by reading code on GitHub, the $\mathrm{R}$ Special Interest Group mailing lists ( $\mathrm{R}$ Core Team, b) such as $r$-sig-mixed-models and r-sig-geo, and other search websites including rpackages.io. 


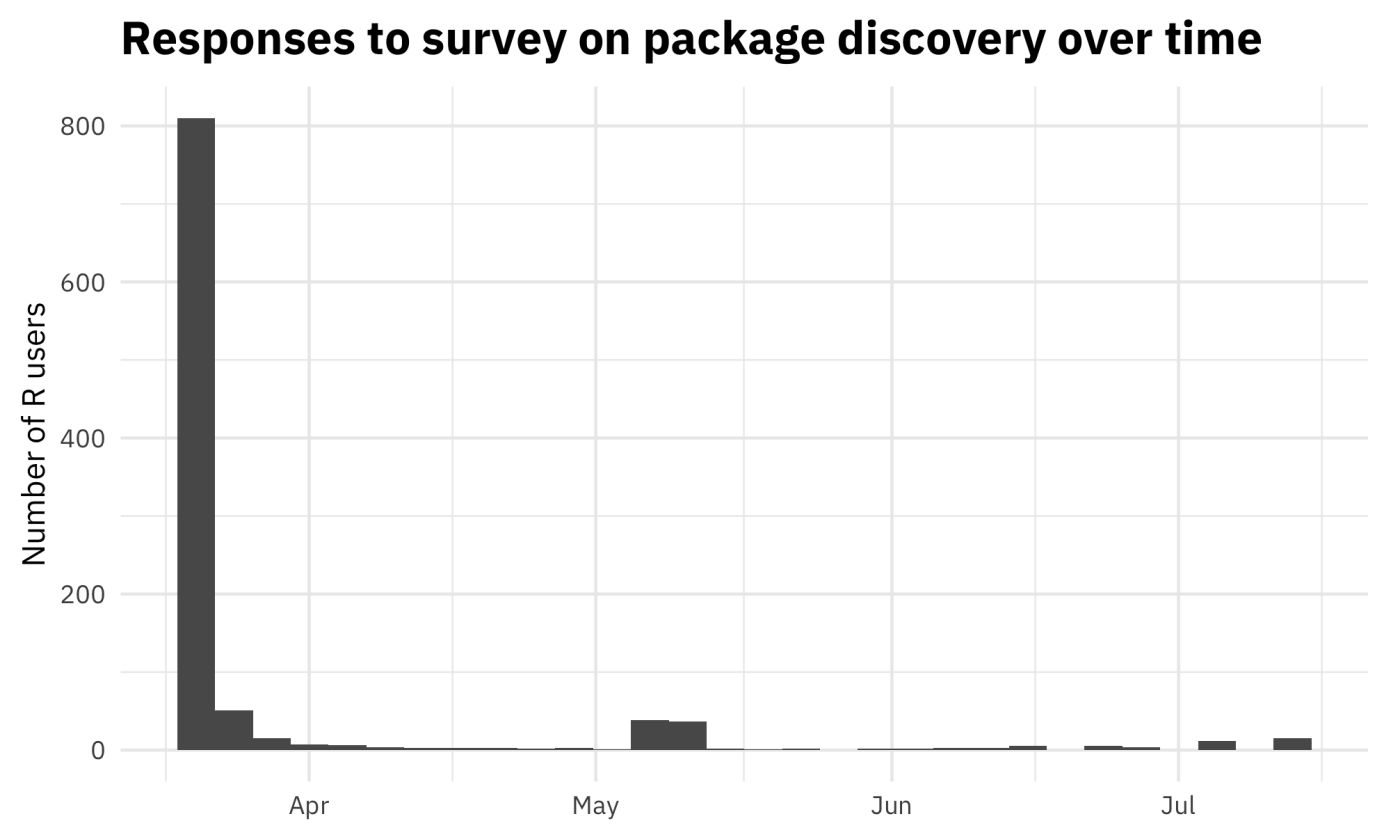

Figure 1: Responses to survey on package discovery during the spring of 2017

In the breakout session at useR!2017 focused on guidance for package choice and package evaluation, we had about 40 participants in our discussion. It was a fruitful discussion and several important themes emerged.

\section{Value of personal impact}

Participants in this session emphasized how impactful personal relationships can be in how packages are shared and evaluated. Some participants discussed how building local networks of $\mathrm{R}$ users may be more important in this effort than top-down, technological solutions. Our survey does show that personal recommendations have been important for many individuals in evaluating $\mathrm{R}$ packages. This is yet another area where local user groups can continue to have important impact. Some ways to share this experience more broadly would be online video series or live data analysis, such as those by Sean Taylor (Taylor, 2017) and Roger Peng (Peng, 2017). Learning through personal networks does not invalidate the importance of other tools like search, especially when it comes to more specialized tasks.

\section{CRAN Task Views}

Some participants wondered whether the idea of a CRAN Task View (Zeileis, 2005) is outdated in the current climate with so many packages, and whether it is even possible for one person to maintain one effectively. In fact, several participants voiced frustration with CTVs that have focused on older packages alone. Others responded that CTVs are focused on curation, which is still important, perhaps even more so now in a world with over 13,000 packages. We had at least one CTV maintainer present in our breakout session, and several things were presented as important in order for CTV maintainers to do their jobs:

- Package maintainers should update their NEWS files.

- Package maintainers need to write good documentation.

These are helpful for all $\mathrm{R}$ users, of course, but also for maintainers of CRAN Task Views. The pkgdown (Wickham and Hesselberth, 2018) package was mentioned as an effective option for making documentation more visible.

\section{CRAN and you}

Participants had several ideas about how things are done on CRAN now and adjustments that might be made in the interest of discovering and evaluating packages. One idea that came up several times was the possibility of keywords or tagging for packages. Since useR!2017, the authors have learned that there is support for some tagging architecture for packages on CRAN in the DESCRIPTION file 
using ACM, JEL, or MSC classifications. For an example of this in action, check out the lfe (Gaure, 2018) package. These are fairly unwieldy lists currently and something like an RStudio addin could be used to navigate them, if they were widely used.

Another desire participants voiced was for more information directly on CRAN, such as the number of downloads for packages. Participants also suggested that vignettes for context-specific tasks like the Bioconductor Workflows (Bioconductor) would be helpful for package discovery and evaluation, either associated with CRAN or perhaps the $R$ Journal. Finally, there was some discussion about whether gate-keeping on CRAN, perceived by most to be reasonable and minimal currently, is good or bad for the community. The conclusion in our discussion was that increased editorial efforts to keep packages off CRAN would not be positive for our community.

\section{More data, more problems}

Some of the package developers at the session wondered why, when $\mathrm{R}$ is a data-centric language, developers have such primitive analytics about their users. Issues of user privacy are central here, but there might be opt-in options that could help both package developers and users make better decisions. The idea of a recommender system for $\mathrm{R}$ packages was brought up multiple times, perhaps a Tinder for R packages like papr, the Tinder for academic preprints (McGowan et al., 2017). Both the users and developers present thought that data on package use (instead of package downloads alone) would be helpful in evaluating how important or helpful $\mathrm{R}$ packages are. Participants also discussed the possibility of a linter for analysis scripts, similar in concept to linters for code (such as Hester (2017)) that automatically analyze code for potential problems and errors, that would suggest packages and good practice. Such a linter would necessarily be opinionated, but almost all efforts to suggest and evaluate $\mathrm{R}$ packages are, by definition.

\section{Unification}

Unification, as we describe it here, attempts to reduce the package count and the span of knowledge required of users. When there are many ways to carry out the same calculation, there are inevitable differences of approach. However, in many respects it is the similarity of approaches that causes most confusion. Very similar calling sequences, unless they are entirely compatible, lead to confusing experiences for users, and threaten the validity of results.

From the experience of one author (JN), the most satisfactory form of unification from the user perspective is the use of wrapper functions that consolidate a number of similar tools into a single calling sequence. This has been the goal of the package optimx, which in its 2018 incarnation consolidates several R-internal and package-based function minimization tools. Moreover, the present version subsumes a number of other packages, thereby offering a reduction in the effective package count.

A real downside of unification is the burden of work for the developers involved, including dealing with complex dependencies and reverse dependencies. At the time of writing, the new optimx fails reverse dependency checks because of new, stricter checks for CRAN policies. Embracing unification as an approach to deal with package proliferation means that such issues are a part of life and must be resolved.

While a wrapper such as optimx can, with effort, be created, merging two existing but different packages that provide similar capability with very different user interfaces requires human cooperation. At this time, and despite the open and collaborative R community, the level of effort to do such work is daunting. Moreover, there is a general lack of financial or other reward for such efforts.

During discussions at useR!2017, it was clear that $\mathrm{R}$ users are quite interested in unification of packages. Younger participants expressed the opinion that there are egos and interest groups standing in the way of some such unifications, and the status of some of the players impeded the discussion of such possibilities.

\section{Conclusion}

Our exploration of these topics leads us to call for increased respect and value for the work done by local meetup group organizers and individuals who contribute to spreading $\mathrm{R}$ knowledge, both online and in their communities. Our survey and discussions show how impactful these community networks are; investing in community building is not something we need do only because of idealism, but because it is effective.

We also identify the importance of growing the skills of package developers across the R ecosystem, in dependency management, documentation, and beyond. Wider adoption of best practices makes the 
$R$ package universe easier for everyone to navigate, from the developer with downstream dependencies to the CRAN Task View maintainer to the new R user.

\section{Bibliography}

J. Baron. Jonathan Baron's R Help Page. Online; accessed 18-November-18. URL http://search . rproject.org/. [p558]

Bioconductor. Bioconductor workflow packages. Online; accessed 18-November-18. URL https: //www. bioconductor.org/packages/release/workflows/. [p561]

J. Cornelissen. RDocumentation: Integrate $R$ with 'RDocumentation', 2018. URL https://CRAN. Rproject. org/package=RDocumentation. R package version 0.8.2. [p558]

G. Csárdi and J. Ooms. METACRAN. Online; accessed 18-November-18. URL https://www.rpkg.org/. [p559]

D. Eddelbuettel. CRANberries. Online; accessed 18-November-18. URL http://dirk. eddelbuettel. com/cranberries/. [p559]

S. Firke, B. Krouse, E. Grand, L. Shepherd, W. Ampeh, and H. Frick. Packagemetrics: Collect Metrics on Packages from CRAN, GitHub, and StackOverflow, 2018. URL https://gi thub.com/ropenscilabs/ packagemetrics. R package version 0.0.1.9001. [p558]

S. Gaure. Ife: Linear Group Fixed Effects, 2018. URL https://CRAN. R-project. org/package=lfe. [p561]

S. Goodman. Rseek search engine. Online; accessed 18-November-18, 2007. URL https://rseek. org/. [p559]

S. Graves. Searching r packages, 2017. URL https://en.wikiversity.org/wiki/Searching_R_ Packages. [p559]

S. Graves. Draft proposal for improving the ability of $r$ users to search $r$ packages, 2018. URL https://en.wikiversity.org/wiki/Draft_Proposal_for_improving_the_ability_ of_R_users_to_search_R_packages. [p559]

S. Graves, S. Dorai-Raj, and R. Francois. Sos: Search Contributed R Packages, Sort by Package, 2017. URL https: //CRAN. R-project.org/package=sos. R package version 2.0-0. [p558]

J. Hester. Lintr: A 'Linter' for R Code, 2017. URL https://CRAN.R-project.org/package=lintr. R package version 1.0.2. [p561]

I. Howson. R package documentation, 2018. URL https://rdrr.io/. [p559]

B. Krouse and A. Calatroni. CRANsearcher: RStudio Addin for Searching Packages in CRAN Database Based on Keywords, 2017. URL https://CRAN. R-project.org/package=CRANsearcher. R package version 1.0.0. [p558]

L. D. McGowan, N. Strayer, and J. Leek. Papr: Tinder for pre-prints, a shiny application for collecting gut-reactions to pre-prints from the scientific community. In The R User Conference, useR! 2017 July 4-7 2017 Brussels, Belgium, page 97, 2017. [p561]

R. Peng. Analyst mindset - episode 2. Online; accessed 18-November-18, 2017. URL https: //youtu . be/jWePleDwmQo. [p560]

R Core Team. Getting Help with R. Online; accessed 18-November-18, a. URL https://www. rproject.org/help.html. [p558]

R Core Team. Mailing Lists. Online; accessed 18-November-18, b. URL https: //www. r-project. org/ mail.htm1. [p559]

R Core Team. Search. Online; accessed 18-November-18, c. URL https: //www. r-project. org/search. html. [p559]

R Core Team. R: A Language and Environment for Statistical Computing. R Foundation for Statistical Computing, Vienna, Austria, 2018. URL https://www.R-project.org/. [p558]

J. Silge. Packagesurvey: Survey of R Users on Package Discovery, 2018. URL https://github.com/ juliasilge/packagesurvey. R package version 0.1.0. [p559] 
S. Taylor. Live estimating nfl statistics. Online; accessed 18-November-18, 2017. URL https://www . facebook.com/seanjtaylor/videos/10103088186201897/. [p560]

H. Wickham and J. Hesselberth. Pkgdown: Make Static HTML Documentation for a Package, 2018. URL https: //CRAN.R-project.org/package=pkgdown. R package version 1.1.0. [p560]

H. Wickham and B. Mæland. crantastic! Online; accessed 18-November-18. URL https://www. crantastic.org/. [p559]

A. Zeileis. CRAN task views. R News, 5(1):39-40, 2005. URL https://CRAN. R-project.org/doc/ Rnews/. [p558, 560]

Julia Silge

Stack Overflow

110 William Street, Floor 28

New York City, NY 10038

julia.silge@gmail. com

John C. Nash

University of Ottawa

Telfer School of Management

Ottawa, ON K1N 6N5, Canada

nashjc@uottawa.ca

Spencer Graves

EffectiveDefense.org

4550 Warwick Blvd. Apt. 508

Kansas City, MO 64111

spencer.graves@effectivedefense.org 